TRANSACTIONS OF THE

AMERICAN MATHEMATICAL SOCIETY

Volume 357, Number 4, Pages 1445-1467

S 0002-9947(04)03522-6

Article electronically published on September 23, 2004

\title{
HYPERPOLYGON SPACES AND THEIR CORES
}

\author{
MEGUMI HARADA AND NICHOLAS PROUDFOOT
}

\begin{abstract}
Given an $n$-tuple of positive real numbers $\left(\alpha_{1}, \ldots, \alpha_{n}\right)$, Konno (2000) defines the hyperpolygon space $X(\alpha)$, a hyperkähler analogue of the Kähler variety $M(\alpha)$ parametrizing polygons in $\mathbb{R}^{3}$ with edge lengths $\left(\alpha_{1}, \ldots, \alpha_{n}\right)$. The polygon space $M(\alpha)$ can be interpreted as the moduli space of stable representations of a certain quiver with fixed dimension vector; from this point of view, $X(\alpha)$ is the hyperkähler quiver variety defined by Nakajima. A quiver variety admits a natural $\mathbb{C}^{*}$-action, and the union of the precompact orbits is called the core. We study the components of the core of $X(\alpha)$, interpreting each one as a moduli space of pairs of polygons in $\mathbb{R}^{3}$ with certain properties. Konno gives a presentation of the cohomology ring of $X(\alpha)$; we extend this result by computing the $\mathbb{C}^{*}$-equivariant cohomology ring, as well as the ordinary and equivariant cohomology rings of the core components.
\end{abstract}

Let $K$ be a compact Lie group acting linearly on $\mathbb{C}^{N}$ with moment map $\mu: \mathbb{C}^{N} \rightarrow \mathfrak{k}^{*}$ such that $\mu(0)=0$. Then for any central regular value $\alpha \in \mathfrak{k}^{*}$, the Kähler quotient

$$
M(\alpha)=\mathbb{C}^{N} / / \alpha=\mu^{-1}(\alpha) / K
$$

is a Kähler manifold of complex dimension $N-\operatorname{dim} K$. The cotangent bundle $T^{*} \mathbb{C}^{N}$ is a hyperkähler manifold, and the induced action of $K$ on $T^{*} \mathbb{C}^{N}$ is hyperhamiltonian (see, for example, $[\mathrm{HP})$, with hyperkähler moment map

$$
\mu_{\mathbb{R}} \oplus \mu_{\mathbb{C}}: T^{*} \mathbb{C}^{N} \rightarrow \mathfrak{k}^{*} \oplus \mathfrak{k}_{\mathbb{C}}^{*}
$$

We call the hyperkähler reduction

$$
X(\alpha)=T^{*} \mathbb{C}^{N} / / / \|_{(\alpha, 0)} K=\left(\mu_{\mathbb{R}}^{-1}(\alpha) \cap \mu_{\mathbb{C}}^{-1}(0)\right) / K
$$

the hyperkähler analogue of $M(\alpha)$. The manifold $X(\alpha)$ is a noncompact hyperkähler manifold of complex dimension $2(N-\operatorname{dim} K)$, containing the cotangent bundle to $M(\alpha)$ as an open set $\left[\mathrm{HP}\right.$. The action of the nonzero complex numbers $\mathbb{C}^{*}$ on $T^{*} \mathbb{C}^{N}$ given by scalar multiplication on each fiber induces an action of $\mathbb{C}^{*}$ on $X(\alpha)$, which restricts to the scalar action on the fibers of $T^{*} M(\alpha) \subseteq X(\alpha)$. The core $\mathfrak{L}$ of $X(\alpha)$ is defined to be the set of points $x \in X(\alpha)$ such that the $\operatorname{limit}_{\lambda \rightarrow \infty} \lambda \cdot x$ exists (the opposite $\operatorname{limit}_{\lambda \rightarrow 0} \lambda \cdot x$ always exists). The action of $\mathbb{C}^{*}$ defines a deformation retraction of $X(\alpha)$ onto $\mathfrak{L}$. If $M(\alpha)$ is nonempty and compact, then $\mathfrak{L}$ is simply the union of all those Białynicki-Birula strata whose closures are compact.

In $[\mathrm{HP}$ we studied the hyperkähler analogues of toric varieties, which arise when $K$ is abelian (see also [BD, HS, [K1]). In this case, $\mathfrak{L}$ is a union of toric varieties, one

Received by the editors August 23, 2003 and, in revised form, October 1, 2003.

2000 Mathematics Subject Classification. Primary 53C26; Secondary 16G20, 14D20.

Key words and phrases. Hyperkähler geometry, polygon space, quiver variety, equivariant cohomology. 
of which is the original toric variety $M(\alpha)$. Another important context in which Kähler reductions and their hyperkähler analogues arise is the study of varieties associated to quivers; this includes the spaces that we will study in this paper.

Suppose a quiver $Q$ (a directed graph) with vertex set $I$ is given, and let $\left\{V_{i} \mid i \in I\right\}$ be a collection of finite complex-dimensional vector spaces. A representation of $Q$ is a collection of maps from $V_{i}$ to $V_{j}$ for every pair of vertices $i$ and $j$ connected by an edge. The group $P U(V)=\left(\prod G L\left(V_{i}\right)\right) / G L(1)_{\Delta}$ acts hamiltonianly on the space $E(Q, V)$ of representations of $Q$. Both the Kähler quotients $M(\alpha)=E(Q, V) \|_{\alpha} P U(V)$ and their hyperkähler analogues $X(\alpha)=T^{*} E(Q, V) / / / \|_{(\alpha, 0)} P U(V)$, called quiver varieties, have been studied extensively. A good introduction to these varieties, both the Kähler and hyperkähler versions, can be found in [N2]. Cores of quiver varieties have attracted particular attention in representation theory. The fundamental classes of their components provide a natural basis for the top homology of $X(\alpha)$, which leads to the construction of canonical bases for representations of the modified universal enveloping algebra associated to the quiver $Q$ [N3] (see also [N4] for more recent results along these lines).

The examples with which we will be concerned in this paper are quiver varieties corresponding to a very special class of quivers, as shown in Figure 1. We label the vertices 0 through $n$, with zero in the center, and for each $i \in\{1, \ldots, n\}$ we have an arrow from $i$ to 0 . We put $V_{i}=\mathbb{C}$ for all $i \in\{1, \ldots, n\}$ and $V_{0}=\mathbb{C}^{2}$, so that

$$
E(Q, V)=\bigoplus_{i=1}^{n} \operatorname{Hom}\left(V_{i}, V_{0}\right) \cong \mathbb{C}^{2 n}
$$

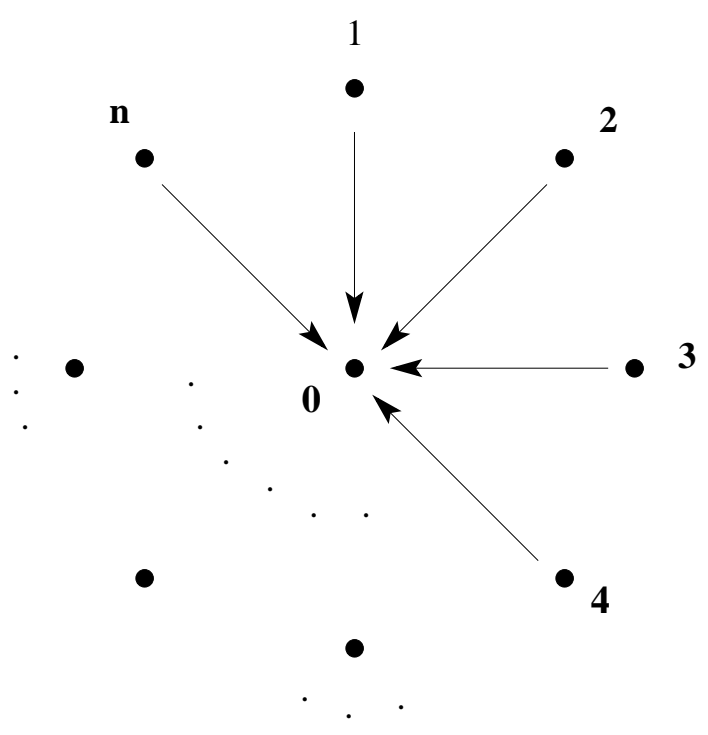

Figure 1. The quiver for hyperpolygon spaces.

In this example, the Kähler quiver variety $M(\alpha)$ has a nice geometric interpretation. The group $P U(V)$ is isomorphic to $\left(S U(2) \times U(1)^{n}\right) / \mathbb{Z}_{2}$, where $\mathbb{Z}_{2}$ acts diagonally 
on the $n+1$ factors, and a central element $\alpha=0 \oplus\left(\alpha_{1}, \ldots, \alpha_{n}\right) \in \mathfrak{s u}(2)^{*} \oplus\left(\mathfrak{u}(1)^{*}\right)^{n}$ is given by an $n$-tuple of real numbers. The variety $M(\alpha)$ is diffeomorphic to the moduli space of $n$-sided polygons in $\mathbb{R}^{3}$, with edge lengths $\left(\alpha_{1}, \ldots, \alpha_{n}\right)$, modulo the action of $S O(3)$ on $\mathbb{R}^{3}$ by rotation [HK1, HK2 $\mathrm{Kl}$, as in Figure 2 .

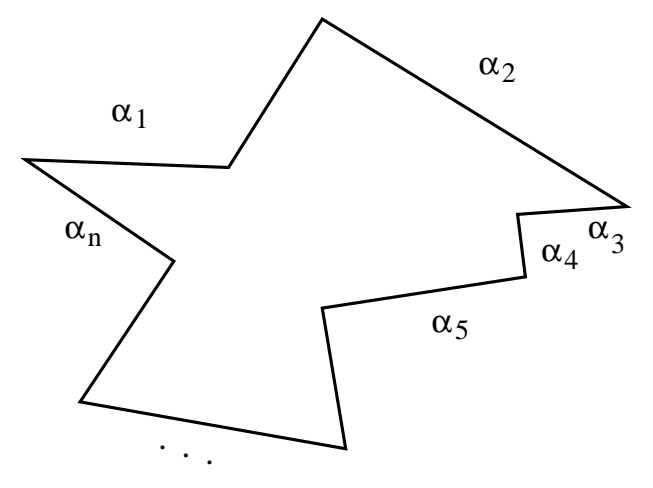

Figure 2. A polygon in $\mathbb{R}^{3}$ with specified edge lengths $\alpha_{i}$.

The hyperkähler analogue $X(\alpha)$ was introduced in [K1], in which Konno enumerated the components of the core of $X(\alpha)$ (Theorem 2.1), showing that the components other than $M(\alpha)$ are in bijection with the collection of subsets $S \subseteq\{1, \ldots, n\}$ of size at least 2 such that $\sum_{i \in S} \alpha_{i}<\sum_{j \in S^{c}} \alpha_{j}$. Such a subset $S$ will be called short. Our first set of results, comprising Section 2 concerns the core components $\left\{U_{S}\right\}$, which one may think of as generalized polygon spaces. We prove that $U_{S}$ is smooth for each short subset $S$ (Theorem 2.2), and interpret it as the moduli space of pairs of polygons in $\mathbb{R}^{3}$ with certain geometric properties (Theorem 2.5). This is therefore a solution, in the special case of polygon spaces, to the following general problem.

Problem 1. Given any moduli space $M$ that can be constructed as a Kähler reduction (or GIT quotient) of complex affine space, is it possible to interpret the core components of the hyperkähler analogue $X$ as moduli spaces corresponding to other related moduli problems?

Our second set of results, comprising Sections 3 and 4 concerns the $S^{1}$-equivariant cohomology of $X(\alpha)$ and $U_{S}$, where $S^{1} \subseteq \mathbb{C}^{*}$ is the unit circle1 Konno computes the cohomology of $X(\alpha)$, showing in particular that the hyperkähler Kirwan map

$$
\kappa: H_{K}^{*}\left(T^{*} \mathbb{C}^{2 n}\right) \rightarrow H_{K}^{*}\left(\mu_{\mathbb{R}}^{-1}(\alpha) \cap \mu_{\mathbb{C}}^{-1}(0)\right) \cong H^{*}(X(\alpha))
$$

in the case of hyperpolygon spaces is surjective (Theorem 3.1). We generalize this result by computing the kernel of the equivariant Kirwan map

$$
\kappa_{S^{1}}: H_{K \times S^{1}}^{*}\left(T^{*} \mathbb{C}^{2 n}\right) \rightarrow H_{K \times S^{1}}^{*}\left(\mu_{\mathbb{R}}^{-1}(\alpha) \cap \mu_{\mathbb{C}}^{-1}(0)\right) \cong H_{S^{1}}^{*}(X(\alpha))
$$

(Theorem 3.2), which is also surjective by Corollary 3.6. In Section 4 we compute the ordinary and equivariant cohomology of a core component $U_{S}$ (Theorem 4.1 and Corollary 4.2). All cohomology rings are taken with coefficients in $\mathbb{Q}$.

\footnotetext{
${ }^{1}$ The $S^{1}$-equivariant cohomology is identical to the $\mathbb{C}^{*}$-equivariant cohomology; we use $S^{1}$ in Section 3 because at times it is convenient to work with the real ADHM description of $X(\alpha)$, in which context the full $\mathbb{C}^{*}$-action is difficult to write down explicitly.
} 
In general, the compactness of the fixed point set $X(\alpha)^{S^{1}}$ gives equivariant cohomology many nice properties not shared by the ordinary cohomology of $X(\alpha)$; for example, the localization theorem of $[\mathrm{AB}]$ makes possible a theory of integration in equivariant cohomology, provided that the fixed point set is compact. Nakajima studies the $S^{1}$-equivariant cohomology and $K$-theory of quiver varieties in [N4, using it to construct representations of quantum affine algebras associated to $Q$. In the process, he conjectures that the equivariant Kirwan map is surjective for all quiver varieties [N4, 7.5.1].

The reader is advised that cohomology computations in hyperpolygon spaces and generalized polygon spaces are often motivated by beautiful and intuitive geometry, but they are just as often driven by daunting, labyrinthine algebra. Whenever possible, we precede the proofs of our theorems with remarks that are aimed at making the geometry maximally transparent.

\section{HyPERPOLYGON SPACES}

We begin by collecting the basic definition and properties of a hyperpolygon space, most of which can be found in $\underline{\mathrm{K} 2}$. Fix a positive integer $n \geq 3$, and consider the group 2

$$
G:=\left(S L(2, \mathbb{C}) \times\left(\mathbb{C}^{*}\right)^{n}\right) / \mathbb{Z}_{2},
$$

where $\mathbb{Z}_{2}$ acts by multiplying each factor by -1 . We define a right action of $G$ on $\mathbb{C}^{2 n}$ as follows. We will write an element of $\mathbb{C}^{2 n}$ as an $n$-tuple of column vectors

$$
q=\left(q_{1}, \ldots, q_{n}\right)
$$

and put

$$
q\left[A ; e_{1}, \ldots, e_{n}\right]=\left(A^{-1} q_{1} e_{1}, \ldots, A^{-1} q_{n} e_{n}\right) .
$$

The compact subgroup

$$
K:=\left(S U(2) \times U(1)^{n}\right) / \mathbb{Z}_{2} \subseteq G
$$

acts with moment map $\mu: \mathbb{C}^{2 n} \rightarrow \mathfrak{s u}(2)^{*} \oplus\left(\mathfrak{u}(1)^{*}\right)^{n}$ given by the equation

$$
\mu(q)=\sum_{i=1}^{n}\left(q_{i} q_{i}^{*}\right)_{0} \oplus\left(\frac{1}{2}\left|q_{1}\right|^{2}, \ldots, \frac{1}{2}\left|q_{n}\right|^{2}\right),
$$

where $q_{i}^{*}$ denotes the conjugate transpose of $q_{i},\left(q_{i} q_{i}^{*}\right)_{0}$ denotes the traceless part of $q_{i} q_{i}^{*}$, and $\mathfrak{s u}(2)^{*}$ is identified with $i \cdot \mathfrak{s u}(2)$ via the trace form. Given an $n$-tuple of real numbers $\left(\alpha_{1}, \ldots, \alpha_{n}\right)$, we define the polygon space

$$
M(\alpha):=\mathbb{C}^{2 n} / /{ }_{\alpha} K=\mu^{-1}(\alpha) / K,
$$

where $\alpha=0 \oplus\left(\alpha_{1}, \ldots, \alpha_{n}\right) \in \mathfrak{s u}(2)^{*} \oplus\left(\mathfrak{u}(1)^{*}\right)^{n}$. If we break the reduction into two steps, reducing first by $U(1)^{n}$ and then by $S U(2)$, we find that

$$
M(\alpha) \cong\left\{\left(v_{1}, \ldots, v_{n}\right) \in\left(\mathbb{R}^{3}\right)^{n} \mid\left\|v_{i}\right\|=\alpha_{i} \text { and } \sum v_{i}=0\right\} / S O(3)
$$

(see Remark 2.6] and the proof of Theorem 2.5). Here $\mathfrak{s u}(2)^{*}$ is being identified with $\mathbb{R}^{3}$, and the coadjoint action of $S U(2)$ on $\mathfrak{s u}(2)^{*}$ is being replaced by the standard action of $S O(3)$ on $\mathbb{R}^{3}$ [HK2]. This space, therefore, may be thought of as the

\footnotetext{
${ }^{2}$ One may prefer to just consider the group $S L(2, \mathbb{C}) \times\left(\mathbb{C}^{*}\right)^{n}$, and allow it to act with a finite kernel. We quotient by $\mathbb{Z}_{2}$ only to be consistent with the conventions of [N2] and [K2].
} 
moduli space of $n$-sided polygons in $\mathbb{R}^{3}$, with fixed edge lengths, up to rotation. In particular, $M(\alpha)$ is empty unless $\alpha_{i} \geq 0$ for all $i$.

We call $\alpha$ generic if there does not exist a subset $S \subseteq\{1, \ldots, n\}$ such that $\sum_{i \in S} \alpha_{i}=\sum_{j \in S^{c}} \alpha_{j}$. Geometrically, this means that there is no element of $M(\alpha)$ represented by a polygon that is contained in a single line in $\mathbb{R}^{3}$. If $\alpha$ is generic, then $M(\alpha)$ is smooth HK1. Throughout this paper we will assume that $\alpha$ is generic, and that $\alpha_{i}>0$ for all $i$.

To define the hyperkähler analogue of $M(\alpha)$, we consider the induced action of $G$ on $T^{*} \mathbb{C}^{2 n}$. Explicitly, we write an element of $T^{*} \mathbb{C}^{2 n}$ as $(p, q)$, where $q=\left(q_{1}, \ldots, q_{n}\right)$ is an $n$-tuple of column vectors and $p=\left(p_{1}, \ldots, p_{n}\right)$ an $n$-tuple of row vectors, and we put

$$
(p, q)\left[A ; e_{1}, \ldots, e_{n}\right]=\left(\left(e_{1}^{-1} p_{1} A, \ldots, e_{n}^{-1} p_{n} A\right),\left(A^{-1} q_{1} e_{1}, \ldots, A^{-1} q_{n} e_{n}\right)\right) .
$$

The vector space $T^{*} \mathbb{C}^{2 n}$ has the structure of a hyperkähler manifold, and the action of $K$ on $T^{*} \mathbb{C}^{2 n}$ is hyperhamiltonian with hyperkähler moment map [K2] (see also [HP])

$$
\mu_{\mathbb{R}} \oplus \mu_{\mathbb{C}}: T^{*} \mathbb{C}^{2 n} \rightarrow\left(\mathfrak{s u}(2)^{*} \oplus\left(\mathfrak{u}(1)^{*}\right)^{n}\right) \oplus\left(\mathfrak{s l}(2, \mathbb{C})^{*} \oplus\left(\mathfrak{u}(1)_{\mathbb{C}}^{n}\right)^{*}\right)
$$

given by the equations

$$
\mu_{\mathbb{R}}(p, q)=\frac{\sqrt{-1}}{2} \sum_{i=1}^{n}\left(q_{i} q_{i}^{*}-p_{i}^{*} p_{i}\right)_{0} \oplus\left(\frac{1}{2}\left(\left|q_{1}\right|^{2}-\left|p_{1}\right|^{2}\right), \ldots, \frac{1}{2}\left(\left|q_{n}\right|^{2}-\left|p_{n}\right|^{2}\right)\right)
$$

and

$$
\mu_{\mathbb{C}}(p, q)=-\sum_{i=1}^{n}\left(q_{i} p_{i}\right)_{0} \oplus\left(\sqrt{-1} p_{1} q_{1}, \ldots, \sqrt{-1} p_{n} q_{n}\right) .
$$

We then define the hyperpolygon space to be the hyperkähler quotient

$$
X(\alpha):=T^{*} \mathbb{C}^{2 n} /\|/\|_{(\alpha, 0)} K=\left(\mu_{\mathbb{R}}^{-1}(\alpha) \cap \mu_{\mathbb{C}}^{-1}(0)\right) / K,
$$

a smooth, noncompact hyperkähler manifold of complex dimension $2(n-3)[\mathrm{K} 2$.

The polygon and hyperpolygon spaces $M(\alpha)$ and $X(\alpha)$ are precisely the Kähler and hyperkähler varieties associated by Nakajima to the quiver shown in Figure 1 It is shown in N1] that

$$
M(\alpha) \cong\left(\mathbb{C}^{2 n}\right)^{\alpha-\mathrm{st}} / G \quad \text { and } \quad X(\alpha) \cong \mu_{\mathbb{C}}^{-1}(0)^{\alpha-\mathrm{st}} / G,
$$

where $\alpha$-st means stable with respect to the weight $\alpha$ in the sense of geometric invariant theory. Nakajima gives a stability criterion for general quiver varieties [N1, [N2], which Konno interprets in the special case of hyperpolygon spaces. Call a subset $S \subseteq\{1, \ldots, n\}$ short if $\sum_{i \in S} \alpha_{i}<\sum_{j \in S^{c}} \alpha_{j}$, and call it long if its complement is short. (Assuming that $\alpha$ is generic is equivalent to assuming that every subset is either short or long.) Given a point $(p, q) \in T^{*} \mathbb{C}^{2 n}$ and a subset $S \subseteq\{1, \ldots, n\}$, we will say that $S$ is straight in $(p, q)$ if $q_{i}$ is proportional to $q_{j}$ for every $i, j \in S$. The terminology comes from Kähler polygon spaces, in which this condition is equivalent to asking that the vectors $v_{i}$ and $v_{j}$ be proportional over $\mathbb{R}_{+}$, or that the edges of lengths $\alpha_{i}$ and $\alpha_{j}$ (if they happen to be adjacent) line up to make a single edge of length $\alpha_{i}+\alpha_{j}$, as in Figure 3 . 


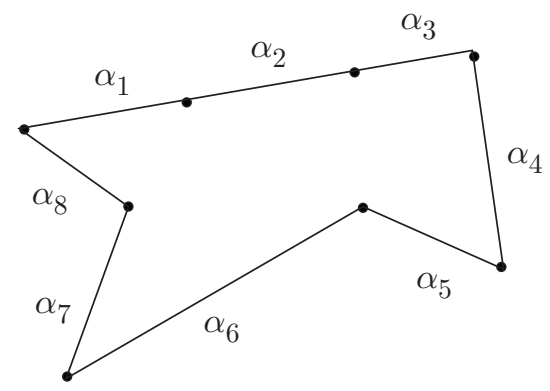

Figure 3 . The subset $\{1,2,3\}$ is straight.

Theorem 1.1 ( $[\mathrm{K2}, 4.2])$. Suppose that $\alpha$ is generic, and $\alpha_{i}>0$ for all $i$. Then a point $(p, q) \in T^{*} \mathbb{C}^{2 n}$ is $\alpha$-stable if and only if the following two conditions are satisfied:

1) $q_{i} \neq 0$ for all $i$, and

2 ) if $S$ is straight and $p_{j}=0$ for all $j \in S^{c}$, then $S$ is short.

We will use the notation $[p, q]$ to denote the $G$-equivalence class of a point $(p, q)$ in $\mu_{\mathbb{C}}^{-1}(0)^{\alpha-s t}$, and $[p, q]_{\mathbb{R}}$ to denote the $K$-equivalence class of a point $(p, q)$ in $\mu_{\mathbb{R}}^{-1}(\alpha) \cap \mu_{\mathbb{C}}^{-1}(0)$. Note that $M(\alpha)$ sits inside of $X(\alpha)$ as the locus of points $[p, q]$ with $p=0$. This observation, along with Theorem 1.1, allows us to recover the $\alpha$-stability condition for the action of $G$ on $\mathbb{C}^{2 n}$. A point $q \in \mathbb{C}^{2 n}$ is $\alpha$-stable if and only if $q_{i} \neq 0$ for all $i$, and no long subset is straight, as first shown in [Kl]. The polygonally-minded reader is warned that in the hyperpolygon space $X(\alpha)$, long subsets can be straight.

\section{THE CORE}

For the rest of the paper we fix a generic $\alpha=0 \oplus\left(\alpha_{1}, \ldots, \alpha_{n}\right) \in \mathfrak{s u}(2)^{*} \oplus\left(\mathfrak{u}(1)^{*}\right)^{n}$, with $\alpha_{i}>0$ for all $i$. To simplify notation, we will write $X=X(\alpha)$ and $M=M(\alpha)$. Consider the action of $\mathbb{C}^{*}$ on $X$ given in the complex description by $\lambda \cdot[p, q]=[\lambda p, q]$. The circle $S^{1} \subseteq \mathbb{C}^{*}$ preserves $\omega_{\mathbb{R}}$, and acts with moment map $\Phi: X \rightarrow \mathbb{R}$ given in the symplectic description by $\Phi\left([p, q]_{\mathbb{R}}\right)=\frac{1}{2} \sum\left|p_{i}\right|^{2}$. Following Konno, we define

$$
\mathcal{S}=\{S \subseteq\{1, \ldots, n\} \mid S \text { is short }\}
$$

and

$$
\mathcal{S}^{\prime}=\{S \in \mathcal{S}|| S \mid \geq 2\} .
$$

Theorem 2.1 ([K2]). The fixed point set $X^{\mathbb{C}^{*}}=X^{S^{1}}=M \cup \bigcup_{S \in \mathcal{S}^{\prime}} X_{S}$, where

$$
X_{S}=\left\{[p, q] \mid S \text { and } S^{c} \text { are each straight, and } p_{j}=0 \text { for all } j \in S^{c}\right\} .
$$

Furthermore, $X_{S}$ is diffeomorphic to $\mathbb{C} P^{|S|-2}$.

For all $S \in \mathcal{S}^{\prime}$, let $U_{S}$ be the closure inside of $X$ of the set

$$
\left\{[p, q] \mid \lim _{\lambda \rightarrow \infty} \lambda \cdot[p, q] \in X_{S}\right\},
$$


and let

$$
\mathfrak{L}=M \cup \bigcup_{S \in \mathcal{S}^{\prime}} U_{S}
$$

This reducible subvariety is called the core of $X$. Since $\lim _{\lambda \rightarrow 0} \lambda \cdot[p, q]$ always exists, the core is simply the union of those Bialynicki-Birula strata whose closures are compact 3 The $\mathbb{C}^{*}$ action defines an $S^{1}$-equivariant deformation retraction of $X$ onto $\mathfrak{L}[\mathrm{N} 1$.

Theorem 2.2. The core component $U_{S}$ is smooth of complex dimension $n-3$, and we have

$$
U_{S}=\left\{[p, q] \mid S \text { is straight, and } p_{j}=0 \text { for all } j \in S^{c}\right\} .
$$

Before proving Theorem 2.2 we describe the way in which the various components of the core fit together. For all $S \in \mathcal{S}^{\prime}$, let

$$
M_{S}=U_{S} \cap M=\{[0, q] \mid S \text { is straight }\} .
$$

We call this space the polygon subspace of $M$ corresponding to the short subset $S$. Note that $M_{S}$ is itself a polygon space with $n-|S|+1$ edges, of lengths $\left\{\alpha_{j} \mid j \in S^{c}\right\} \cup\left\{\sum_{S} \alpha_{i}\right\}$. In particular, it is smooth. Now suppose given two short subsets $S, T \in \mathcal{S}^{\prime}$, and consider the intersection $U_{S} \cap U_{T}$.

- If $S \cap T=\emptyset$, then $U_{S} \cap U_{T}=M_{S} \cap M_{T}$, a polygon subspace both of $M_{S}$ and of $M_{T}$.

- If $S \cap T \neq \emptyset$ and $S \cup T$ is long, then $U_{S} \cap U_{T}=\emptyset$.

- If $S \cap T \neq \emptyset$ and $S \cup T$ is short, then

$$
U_{S} \cap U_{T}=\left\{\begin{array}{l|l}
{[p, q]} & \begin{array}{l}
S \cup T \text { is straight, } \\
p_{j}=0 \text { for all } j \in(S \cap T)^{c}
\end{array}
\end{array}\right\} .
$$

This is a subvariety of $U_{S \cup T}$ given by taking the closure inside of $U_{S \cup T}$ of a certain subbundle of the conormal bundle to $M_{S \cup T} \subseteq M$, defined by setting $p_{j}=0$ for all $j \in(S \cap T)^{c} \supseteq(S \cup T)^{c}$.

Each of these descriptions generalizes to higher intersections without modification.

Finally, we compute the fixed point set $U_{S}^{\mathbb{C}^{*}}$. If $[p, q] \in U_{S}^{\mathbb{C}^{*}}$, then either $p=0$ and $[p, q] \in M_{S}$, or $[p, q] \in X_{T}$ for some $T \in \mathcal{S}^{\prime}$. If $[p, q] \in X_{T}$ then Theorem 2.1 tells us that $T$ and $T^{c}$ are each straight, hence $S \subseteq T$ or $S \subseteq T^{c}$. Since $p \neq 0$, we must have $S \subseteq T$. Indeed, $U_{S} \cap X_{T}$ is the linear subspace of $X_{T} \cong \mathbb{C} P^{|T|-2}$ given by the condition $p_{j}=0$ for all $j \in T \backslash S$. In particular, $U_{S} \cap X_{T}$ is isomorphic to $\mathbb{C} P^{|S|-2}$ for any $T \supseteq S$.

Example 2.3. Let $n=5, \alpha_{1}=\alpha_{2}=1$, and $\alpha_{3}=\alpha_{4}=\alpha_{5}=3$, and consider the short subset $S=\{1,2\}$. The fixed point set of $U_{S}$ consists of $M_{S} \cong \mathbb{C} P^{1}$, and four points $X_{S}, U_{S} \cap X_{T_{3}}, U_{S} \cap X_{T_{4}}$, and $U_{S} \cap X_{T_{5}}$, where $T_{j}=\{1,2, j\}$ for $j=3,4,5$. For each $j, U_{S} \cap U_{T_{j}}$ is isomorphic to $\mathbb{C} P^{1}$, and touches $M_{S}$ at the point $M_{T_{j}}$. In the following picture, an ellipse represents a copy of $\mathbb{C} P^{1}$ flowing between two fixed points, where the numbers or pairs of numbers indicate subsets that are straight on this $\mathbb{C} P^{1}$. (For example, 12, 45 means that 1 and 2 are straight, as are 4 and 5.) We will revisit this example at the end of Section 4

\footnotetext{
${ }^{3}$ In Morse theoretic language, $U_{S}$ is the closed flow-down set for $X_{S}$ with respect to the MorseBott function $\Phi$, and $C$ is the union of all of the flow-down sets.
} 


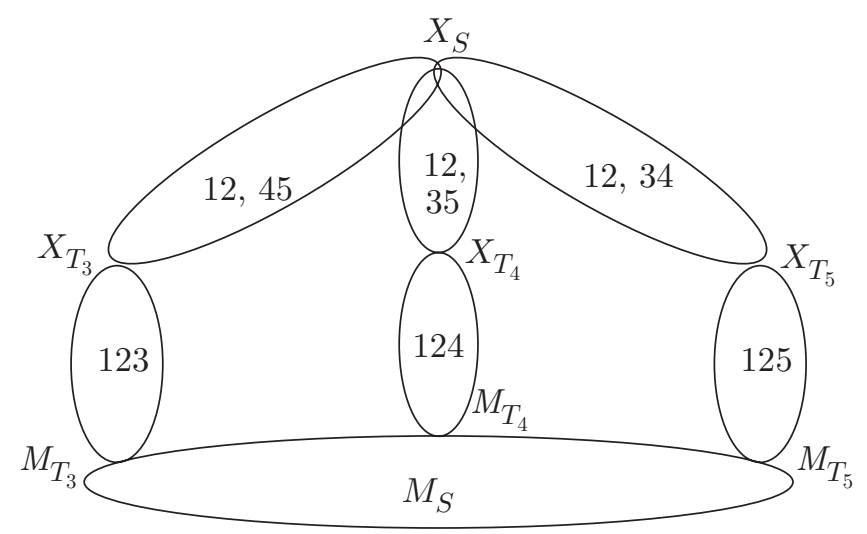

Figure 4. $U_{S}$, with $S=\{1,2\}$

Proof of Theorem 2.2. The fact that $\operatorname{dim} U_{S}=\frac{1}{2} \operatorname{dim} X=n-3$ is a general property of core components of quiver varieties [N1]. Thus, by a dimension count, it is enough to show that the set $\left\{[p, q] \mid S\right.$ is straight, and $p_{j}=0$ for all $\left.j \in S^{c}\right\}$ is contained in $U_{S}$.

Consider a point $[p, q] \in X$ with $S$ straight, and $p_{j}=0$ for all $j \in S^{c}$. By applying an element of $G$, we may assume that $q_{i}=\left(\begin{array}{l}1 \\ 0\end{array}\right)$ for all $i \in S$. Suppose further that there exists an $i \in S$ with $p_{i} \neq 0$, and that no strict superset of $S$ is straight. In other words, if $q_{j}=\left(\begin{array}{c}a_{j} \\ b_{j}\end{array}\right)$ for $j \in S^{c}$, suppose that $b_{j} \neq 0$. For $\lambda \in \mathbb{C}^{*}$, let $A(\lambda)=\left(\begin{array}{cc}\lambda & 0 \\ 0 & \lambda^{-1}\end{array}\right)$, let $e_{i}(\lambda)=\lambda$ for all $i \in S$, and let $e_{j}(\lambda)=\lambda^{-1}$ for all $j \in S^{c}$. Then for $i \in S$, we have $e_{i}(\lambda)^{-1} p_{i} A(\lambda)=\lambda^{-2} p_{i}$ and $A(\lambda)^{-1} q_{i} e_{i}=q_{i}$. For $j \in S^{c}$, we have $A(\lambda)^{-1} q_{j} e_{j}=\left(\begin{array}{c}\lambda^{-2} a_{j} \\ b_{j}\end{array}\right)$. Hence

$$
\begin{aligned}
\lim _{\lambda \rightarrow \infty} \lambda \cdot[p, q] & =\lim _{\lambda \rightarrow \infty} \lambda^{2} \cdot[p, q] \\
& =\lim _{\lambda \rightarrow \infty}\left[\lambda^{2} p, q\right] \\
& =\lim _{\lambda \rightarrow \infty}\left[\lambda^{2} e(\lambda)^{-1} p A(\lambda), A(\lambda)^{-1} q e(\lambda)\right] \\
& =\left[p, q^{\prime}\right],
\end{aligned}
$$

where $q_{i}^{\prime}=q_{i}$ for $i \in S$, and $q_{j}^{\prime}=\left(\begin{array}{c}0 \\ b_{j}\end{array}\right)$ for $j \in S^{c}$. Since we have assumed that $b_{j} \neq 0$ for all $j \in S^{c}$ and that $p_{i} \neq 0$ for some $i \in S,\left(p, q^{\prime}\right)$ is stable, and hence defines an element of $X_{S}$. Since $U_{S}$ is defined to be the closure of the set of elements that flow up to $X_{S}$, it includes all $[p, q]$ with $S$ straight and $p_{j}=0$ for all $j \in S^{c}$.

To see that $U_{S}$ is smooth, it is sufficient to show that $U_{S}$ is smooth at $[p, q]$ for all $[p, q] \in X^{\mathbb{C}^{*}}$. First suppose that $[p, q] \in X_{T}$ for some $T \in \mathcal{S}^{\prime}$ containing $S$. Suppose, without loss of generality, that $T=\{1, \ldots, l\}$ and $S=\{1, \ldots, m\}$ for some $l \leq m$. Konno computes an explicit local complex chart for $X$ at the point $[p, q]$, with coordinates $\left\{z_{i}, w_{i} \mid 3 \leq i \leq n-1\right\}$ [K2. With respect to these coordinates, a point $\left[p^{\prime}, q^{\prime}\right]$ has the property that $S$ is straight and $p_{j}^{\prime}=0$ for all $j \in S^{c}$ if and only if $w_{i}=0$ for all $3 \leq i \leq l$ and $z_{j}=0$ for all $l+1 \leq j \leq n-1$. Hence $U_{S}$ is smooth at $[p, q]$. 
It remains only to show that $U_{S}$ is smooth at $M_{S}=U_{S} \cap M$. Let

$$
E=\left\{(p, q) \mid S \text { is straight, } p_{j}=0 \text { for all } j \in S^{c} \text {, and } \mu_{\mathbb{C}}(p, q)=0\right\},
$$

and let $N=\{(p, q) \in E \mid p=0\}$. The natural projection from $E$ to $N$ exhibits $E$ as a vector bundle over $N$, because the equation $\mu_{\mathbb{C}}(p, q)=0$ is linear in $p$. By definition, $U_{S}=E / / G=E^{\alpha-\mathrm{st}} / G$, and $M_{S}=N / / G=N^{\alpha-\mathrm{st}} / G$. The set $\left.E\right|_{N^{\alpha-s t}} / G \subseteq E^{\alpha-s t} / G$ is an open neighborhood of $M_{S}$ inside of $U_{S}$, and is isomorphic to a vector bundle over $M_{S}$. Since $M_{S}$ is a polygon space it is smooth, hence $U_{S}$ is smooth in a neighborhood of $M_{S}$.

The following corollary is known to the experts; we include it here for lack of an explicit reference.

Corollary 2.4. $U_{S}$ is a compactification of the conormal bundle to $M_{S}$ in $M$.

Proof. We must show that the normal bundle to $M_{S}$ in $U_{S}$ is dual to the normal bundle to $M_{S}$ in $M$. We use only general facts about quiver varieties from [N1], and the additional information that $U_{S}$ is smooth, from Theorem 2.2. Consider a point $[0, q] \in M_{S}$, and let $H_{0}$ and $H_{1}$ be the 0 and 1 weight spaces of the $\mathbb{C}^{*}$ action on $T_{[0, q]} X$. The holomorphic symplectic form $\omega_{\mathbb{C}}$ is being rotated by $\mathbb{C}^{*}$ with weight 1 [N1, 5.1], hence it defines a perfect pairing between $H_{0}$ and $H_{1}$. The fiber at $[0, q]$ of the normal bundle to $M_{S}$ in $M$ is $H_{0} / T_{[0, q]} M_{S}$, which is dual by $\omega_{\mathbb{C}}$ to the annihilator of $T_{[0, q]} M_{S}$ inside of $H_{1}$. Since $U_{S}$ is $\mathbb{C}^{*}$-invariant, we may write

$$
T_{[0, q]} U_{S}=T_{[0, q]} U_{S} \cap H_{0} \oplus T_{[0, q]} U_{S} \cap H_{1}=T_{[0, q]} M_{S} \oplus T_{[0, q]} U_{S} \cap H_{1} .
$$

To prove Corollary 2.4, we must show that $T_{[0, q]} U_{S} \cap H_{1}$ is equal to the annihilator of $T_{[0, q]} M_{S}$. The fact that $T_{[0, q]} U_{S} \cap H_{1}$ is contained in the annihilator of $T_{[0, q]} M_{S}$ follows from the fact that $U_{S}$ is lagrangian with respect to $\omega_{\mathbb{C}}$ [N1] (here we use smoothness of $U_{S}$ at $\left.[0, q]\right)$. Equality is then obtained by dimension count.

We next describe $U_{S}$ in polygon-theoretic terms, as a certain moduli space of pairs of polygons in $\mathbb{R}^{3}$.

Theorem 2.5. Let $U_{S}$ be the component of the core of $X$ corresponding to a subset $S \in \mathcal{S}^{\prime}$. Then $U_{S}$ is homeomorphic to the moduli space of $n+1$ vectors

$$
\left\{u_{i}, v_{j}, w \in \mathbb{R}^{3} \mid i \in S, j \in S^{c}\right\},
$$

taken up to rotation, satisfying the following conditions:

$$
\begin{array}{ll}
\text { 1) } & w+\sum_{j \in S^{c}} v_{j}=0, \\
\text { 2) } & \sum_{i \in S} u_{i}=0, \\
\text { 3) } & u_{i} \cdot w=0 \quad \text { for all } i \in S, \\
\text { 4) } & \left\|v_{j}\right\|=\alpha_{j} \quad \text { for all } j \in S^{c}, \\
\text { 5) } & \|w\|=\sum_{i \in S} \sqrt{\alpha_{i}^{2}+\left\|u_{i}\right\|^{2}} .
\end{array}
$$

Remark 2.6. In more descriptive terms, a point in $U_{S}$ specifies two polygons in $\mathbb{R}^{3}$, as in Figure [5] The first is the $n-|S|+1$-sided polygon consisting of the vectors $\left\{v_{j} \mid j \in S^{c}\right\}$ and $w$. Each vector $v_{j}$ has length $\alpha_{j}$, and $w$ has a variable length, always greater than or equal to $\sum_{i \in S} \alpha_{i}$. This variable length is determined by the 


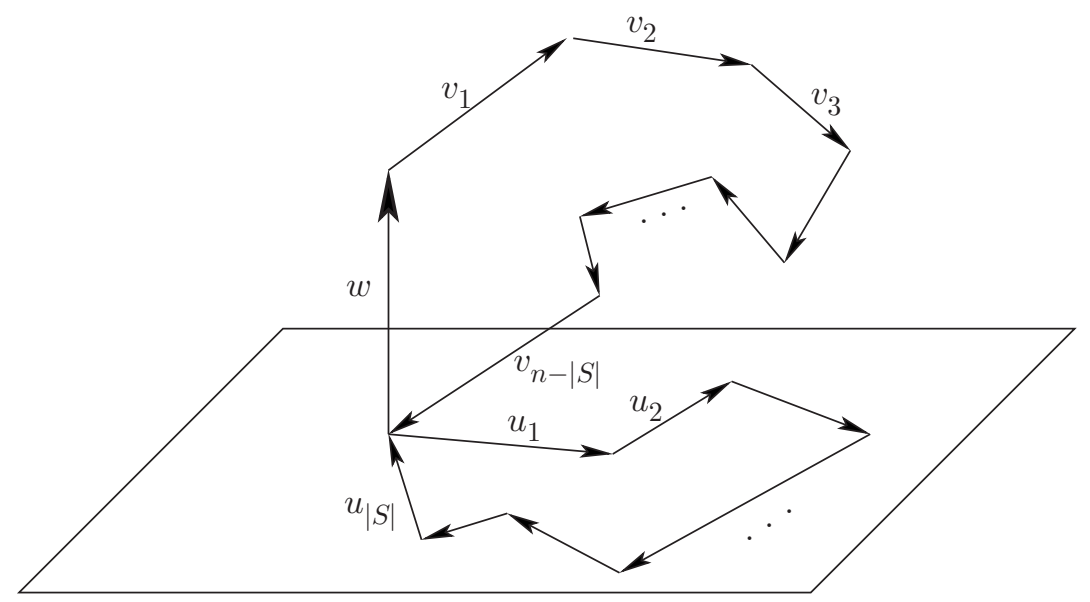

Figure 5. An element of $U_{S}$, represented by a spatial polygon with a distinguished edge, and a planar polygon perpendicular to that edge.

lengths of the edges in the second polygon, which consists of $|S|$ vectors $\left\{u_{i} \mid i \in S\right\}$, all contained in the plane perpendicular to $w$. Note that this description also applies to the Kähler polygon space $M$ by taking $S=\emptyset$.

By setting $u_{i}=0$ for all $i$ we get $M_{S}$, the minimum of the Morse-Bott function $\Phi$ on $U_{S}$. On the other hand, consider the submanifold of $U_{S}$ obtained by imposing the extra condition that $\|w\|=\sum_{j \in S^{c}}\left\|v_{j}\right\|$. Then the first of the two polygons is forced to be linear, and we are left with $|S|$ vectors $\left\{u_{i}\right\}$ in the perpendicular plane satisfying a certain norm condition and adding to zero. Identifying this plane with $\mathbb{C}$ and dividing by the circle action rotating this plane, we obtain $\mathbb{C} P^{|S|-2} \cong X_{S}$, the maximum of $\Phi$ on $U_{S}$. Other critical points of $\Phi$ occur whenever the first polygon is linear, which is possible for finitely many values of $\|w\|$.

Proof of [2.5. Suppose a point $[p, q]_{\mathbb{R}} \in U_{S}$ is given, and let

$$
\begin{gathered}
u_{i}=q_{i} p_{i}+p_{i}^{*} q_{i}^{*} \quad \text { for all } i \in S, \\
v_{j}=\left(q_{j} q_{j}^{*}\right)_{0} \quad \text { for all } j \in S^{c}, \\
w=\sum_{i \in S}\left(q_{i} q_{i}^{*}\right)_{0}-\left(p_{i}^{*} p_{i}\right)_{0} .
\end{gathered}
$$

These vectors live in $i \cdot \mathfrak{s u}(2) \cong \mathfrak{s u}(2)^{*} \cong \mathbb{R}^{3}$, which is endowed with the metric $A \cdot B=\frac{1}{2} \operatorname{tr} A B$, invariant under the coadjoint action. With respect to this metric, we have the equalities $\left\|\left(q q^{*}\right)_{0}\right\|=\frac{1}{2}|q|^{2}$ and $\left\|\left(p^{*} p\right)_{0}\right\|=\frac{1}{2}|p|^{2}$, hence conditions (1), (2), and (4) are immediate consequences of the moment map equations.

To verify condition (3), note that the vectors $\left\{q_{i} \mid i \in S\right\}$ are all proportional over $\mathbb{C}$, which implies that the vectors $\left(q_{i} q_{i}^{*}\right)_{0}$ are positive scalar multiples of each other. Furthermore, the moment map equation $p_{i} q_{i}=0$ implies that $\left(p_{i}^{*} p_{i}\right)_{0}$ is a nonpositive scalar multiple of $\left(q_{i} q_{i}^{*}\right)_{0}$, therefore $w=\sum\left(q_{i} q_{i}^{*}\right)_{0}-\left(p_{i}^{*} p_{i}\right)_{0}$ is proportional over $\mathbb{R}_{+}$to $\left(q_{i} q_{i}^{*}\right)_{0}$ for any $i \in S$. Then $u_{i} \cdot w=\frac{1}{2} \operatorname{tr} u_{i} w$ is a multiple of

$$
\operatorname{tr} u_{i}\left(q_{i} q_{i}^{*}\right)_{0}=\operatorname{tr} u_{i} q_{i} q_{i}^{*}=\operatorname{tr} p_{i}^{*} q_{i}^{*} q_{i} q_{i}^{*}=\left|q_{i}\right|^{2} \operatorname{tr} p_{i}^{*} q_{i}^{*}=0,
$$


where the first equality comes from the fact that $q_{i} q_{i}^{*}-\left(q_{i} q_{i}^{*}\right)_{0}$ is a scalar multiple of the identity, and $\operatorname{tr} u_{i}=0$.

To check condition (5), we first compute the norm of $u_{i}$ :

$$
\begin{aligned}
\left\|u_{i}\right\|^{2} & =\frac{1}{2} \operatorname{tr} u_{i}^{2} \\
& =\frac{1}{2} \operatorname{tr}\left(q_{i} p_{i} p_{i}^{*} q_{i}^{*}+p_{i}^{*} q_{i}^{*} q_{i} p_{i}\right) \\
& =\left|q_{i}\right|^{2}\left|p_{i}\right|^{2} \\
& =\left|q_{i}\right|^{2}\left(\left|q_{i}\right|^{2}-2 \alpha_{i}\right) .
\end{aligned}
$$

Since all of the vectors $\left\{\left(q_{i} q_{i}^{*}\right)_{0}-\left(p_{i}^{*} p_{i}\right)_{0} \mid i \in S\right\}$ point in the same direction, we have

$$
\begin{aligned}
\|w\| & =\sum_{i \in S}\left\|\left(q_{i} q_{i}^{*}\right)_{0}\right\|+\left\|\left(p_{i}^{*} p_{i}\right)_{0}\right\|=\sum_{i \in S} \frac{1}{2}\left|q_{i}\right|^{2}+\frac{1}{2}\left|p_{i}\right|^{2} \\
& =\sum_{i \in S}\left|q_{i}\right|^{2}-\alpha_{i}=\sum_{i \in S} \sqrt{\alpha_{i}^{2}+\left\|u_{i}\right\|^{2}}
\end{aligned}
$$

We have defined a map from $U_{S}$ to the moduli space of vectors $\left\{u_{i}, v_{j}, w\right\}$ satisfying conditions (1)-(5), and we claim that this map is a homeomorphism. Since the source of this map is compact and the target is Hausdorff, it is sufficient to show that the map is bijective.

Suppose a collection of vectors $\left\{u_{i}, v_{j}, w\right\} \subseteq \mathfrak{s u}(2)$ satisfying conditions (1)-(5) is given. Using the adjoint action of $S U(2)$, we may assume that $w$ is a positive scalar multiple of $\left(\begin{array}{cc}1 & 0 \\ 0 & -1\end{array}\right)$. By condition (3), this implies that for all $i \in S$, there exists $\lambda_{i} \in \mathbb{C}$ with $u_{i}=\left(\begin{array}{cc}0 & \lambda_{i} \\ \bar{\lambda}_{i} & 0\end{array}\right)$. For $j \in S^{c}$, we choose $q_{j} \in \mathbb{C}^{2}$ with $\left(q_{j} q_{j}^{*}\right)_{0}=v_{j}$, and observe that $q_{j}$ is unique up to the action of $U(1)^{n}$. We know that for all $i \in S$, $\left(q_{i} q_{i}^{*}\right)_{0}$ must be a positive multiple of $w$, hence there exist $a_{i}, b_{i} \in \mathbb{C}$ such that

$$
q_{i}=\left(\begin{array}{c}
a_{i} \\
0
\end{array}\right) \text { and } p_{i}=\left(\begin{array}{ll}
0 & b_{i}
\end{array}\right)
$$

for all $i \in S$. In order to have $u_{i}=q_{i} p_{i}+p_{i}^{*} q_{i}^{*}$ and $\frac{1}{2}\left|q_{i}\right|^{2}-\frac{1}{2}\left|p_{i}\right|^{2}=\alpha_{i}$, we must have

$$
a_{i} b_{i}=\lambda_{i} \text { and } \frac{1}{2}\left|a_{i}\right|^{2}-\frac{1}{2}\left|b_{i}\right|^{2}=\alpha_{i}
$$

These equations uniquely define $a_{i}$ and $b_{i}$ up to the action of $U(1)^{n}$. It follows from conditions (1)-(5) that $(p, q) \in \mu_{\mathbb{R}}^{-1}(\alpha) \cap \mu_{\mathbb{C}}^{-1}(0)$ and that $w=\sum_{i \in S}\left(q_{i} q_{i}^{*}\right)_{0}-\left(p_{i}^{*} p_{i}\right)_{0}$. This shows that our map is bijective, and thus completes the proof of Theorem 2.5 .

Remark 2.7. Suppose that $S$ has only two elements; without loss of generality we will assume that $S=\{1,2\}$. Then forgetting $u_{1}$ and $u_{2}$ gives a diffeomorphism from $U_{S}$ to the "vertical polygon space" $V P\left(\alpha_{3}, \ldots, \alpha_{n}, \alpha_{1}+\alpha_{2}\right)$ defined in [HK2], shown to be diffeomorphic to a toric variety. More generally with $S=\{1, \ldots, k\}$, given any two-element subset $T \subseteq S$, the subvariety of $U_{S}$ given by the equations $u_{i}=0$ for all $i \in S \backslash T$ is diffeomorphic to $V P\left(\alpha_{k+1}, \ldots, \alpha_{n}, \sum_{T} \alpha_{i}\right)$. 


\section{Equivariant cohomology of $\mathbf{X}$}

We begin by defining representations

$$
\rho_{i j}: K \rightarrow U(1) \quad \text { and } \quad \rho_{S O(3)}: K \rightarrow S O(\mathfrak{s u}(2))
$$

by the formulae

$$
\rho_{i j}\left[A ; e_{1}, \ldots, e_{n}\right]=e_{i} e_{j} \quad \text { and } \quad \rho_{S O(3)}\left[A ; e_{1}, \ldots, e_{n}\right]=\operatorname{Ad}(A) .
$$

Associated to these representations are the vector bundles

$$
\mathcal{L}_{i j}=\left(\mu_{\mathbb{R}}^{-1}(\alpha) \cap \mu_{\mathbb{C}}^{-1}(0)\right) \times_{\rho_{i j}} \mathbb{C} \quad \text { and } \quad \mathcal{E}=\left(\mu_{\mathbb{R}}^{-1}(\alpha) \cap \mu_{\mathbb{C}}^{-1}(0)\right) \times_{\rho_{S O(3)}} \mathfrak{s u}(2) .
$$

Let $c_{i}=c_{1}\left(\mathcal{L}_{i i}\right) \in H^{2}(X)$ and $p=p_{1}(\mathcal{E}) \in H^{4}(X)$.

Theorem 3.1 (Konno [K2]). The cohomology ring $H^{*}(X)$ is isomorphic to $\mathbb{Q}\left[c_{1}, \ldots, c_{n}, p\right] / \mathcal{I}$, where $\mathcal{I}$ is generated by the following two families:

1) $p-c_{i}^{2}$ for all $i \in\{1, \ldots, n\}$,

2) all elements of degree $2(n-2)$.

The action of $S^{1}$ on the total spaces of $\mathcal{L}_{i j}$ and $\mathcal{E}$ given by the left action on $\mu_{\mathbb{R}}^{-1}(\alpha) \cap \mu_{\mathbb{C}}^{-1}(0)$ gives these bundles an $S^{1}$-equivariant structure. For the rest of this section, we will use $c_{i}$ and $p$ to denote the equivariant characteristic classes

$$
c_{i}=c_{1}\left(\mathcal{L}_{i i}\right) \in H_{S^{1}}^{2}(X) \quad \text { and } \quad p=p_{1}(\mathcal{E}) \in H_{S^{1}}^{4}(X) .
$$

Let $\mathcal{K}$ be the $S^{1}$-equivariant line bundle on $X$ obtained by pulling back the weight 1 line bundle over a point, and let

$$
x=c_{1}(\mathcal{K}) \in H_{S^{1}}^{2}(X) .
$$

We obtain the following result, extending Konno's work to the equivariant context.

Theorem 3.2. The equivariant cohomology ring $H_{S^{1}}^{*}(X)$ is isomorphic to $\mathbb{Q}\left[c_{1}, \ldots, c_{n}, p, x\right] / \mathcal{J}$, where $\mathcal{J}$ is generated by the following two families:

$$
\begin{aligned}
& \text { 1) } \quad p-c_{i}^{2} \text { for all } i \in\{1, \ldots, n\}, \\
& \prod_{j \in \overline{S^{c}}}\left(c_{j}+c_{n_{S}}\right) \times \prod_{i \in \bar{S}}\left(c_{i}+x\right) \text { for all } \emptyset \neq S \in \mathcal{S},
\end{aligned}
$$

where $m_{S} \in S$ and $n_{S} \in S^{c}$ are the minimal elements of the two sets, $\bar{S}=S \backslash\left\{m_{S}\right\}$, and $\overline{S^{c}}=S^{c} \backslash\left\{n_{S}\right\}$.

Remark 3.3. Konno observes that the quotient map from the abstract polynomial ring $\mathbb{Q}\left[c_{1}, \ldots, c_{n}, p\right]$ to $H^{*}(X)$ is precisely the Kirwan map

$$
\kappa: H_{K}^{*}\left(T^{*} \mathbb{C}^{2 n}\right) \rightarrow H^{*}(X)
$$

induced by the inclusion $\mu_{\mathbb{R}}^{-1}(\alpha) \cap \mu_{\mathbb{C}}^{-1}(0) \hookrightarrow T^{*} \mathbb{C}^{2 n}$. Theorem 3.1 can be interpreted as saying that the Kirwan map for hyperpolygon spaces is surjective, with kernel $\mathcal{I}$. Likewise, Theorem 3.2 asserts that the $S^{1}$-equivariant Kirwan map

$$
\kappa_{S^{1}}: H_{K \times S^{1}}^{*}\left(T^{*} \mathbb{C}^{2 n}\right) \rightarrow H_{S^{1}}^{*}(X)
$$

is surjective, with kernel $\mathcal{J}$. The analogous map for Kähler reductions is known to always be surjective [Ki], but in the hyperkähler case the problem remains open. 
Remark 3.4. The second family of relations in Theorem 3.2 has a geometric interpretation. In the course of the proof of Theorem 3.2 it will be shown that the class $-\frac{1}{2}\left(c_{j}+c_{n_{s}}\right)$ is represented by the divisor given by the points $[p, q] \in X$, where $q_{j}$ and $q_{n_{s}}$ are straight. Hence the product $\prod_{i \in \overline{S^{c}}}\left(c_{j}+c_{n_{s}}\right)$ is supported on the subvariety of points, where $S^{c}$ is straight. Similarly, it is shown in the proof that the class $c_{i}+x$ is represented by the divisor given by the condition $p_{i}=0$, hence the class

$$
\prod_{j \in \bar{S}^{c}}\left(c_{j}+c_{n_{S}}\right) \times \prod_{i \in \bar{S}}\left(c_{i}+x\right)
$$

is supported on the subvariety of points $[p, q]$, where a long subset $S^{c}$ is straight, and all but one $p_{i}$ for $i \in S$ is zero. We will show, using Theorem 2.1, that this subvariety is disjoint from the fixed point set $X^{S^{1}}$, and that this implies that its cohomology class is trivial.

Before proceeding with the proof of Theorem 3.2, we collect some preliminary results regarding the relationship between ordinary and equivariant cohomology. The first result that we need is known as equivariant formality, proven for compact manifolds in Ki], which we adapt to our situation in Proposition 3.5.

Proposition 3.5. Let $X$ be a symplectic manifold, possibly noncompact but of finite topological type. Suppose that $X$ admits a hamiltonian circle action, and that the moment map is proper and bounded below. Then $H_{S^{1}}^{*}(X)$ is a free module over $H_{S^{1}}^{*}(p t)$.

Proof. Because $\Phi$ is a moment map, it is a Morse-Bott function such that all of the critical submanifolds and their normal bundles carry almost complex structures. Thus we get a Morse-Bott decomposition of $X$ into even-dimensional $S^{1}$-invariant submanifolds. This tells us, as in [Ki], that the spectral sequence associated to the fibration $X \hookrightarrow E G \times_{G} X \rightarrow B G$ collapses, and we get the desired result.

Consider the following commuting square of maps, where $\phi$ and $\psi$ are each given by setting $x$ to zero.

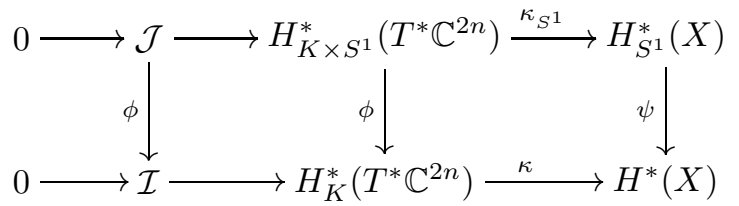

Our moment map $\Phi: X \rightarrow \mathbb{R}$ is proper and bounded below [HP, 1.3], therefore Proposition 3.5 has the following consequences.

Corollary 3.6. The equivariant Kirwan map $\kappa_{S^{1}}$ is surjective.

Proof. Suppose that $\gamma \in H_{S^{1}}^{*}(X)$ is a homogeneous class of minimal degree that is not in the image of $\kappa_{S^{1}}$, and choose a class $\eta \in \phi^{-1} \kappa^{-1} \psi(\gamma)$. Then by Proposition 3.5, $\kappa_{S^{1}}(\eta)-\gamma=x \delta$ for some $\delta \in H_{S^{1}}^{*}(M)$, and therefore $\delta$ is a class of lower degree that is not in the image of $\kappa_{S^{1}}$.

Corollary 3.7. Suppose that $\mathcal{J} \subseteq \operatorname{ker} \kappa_{S^{1}}$ and $\phi(\mathcal{J})=\mathcal{I}$. Then $\mathcal{J}=\operatorname{ker} \kappa_{S^{1}}$.

Proof. Suppose that $a \in \operatorname{ker} \kappa_{S^{1}} \backslash \mathcal{J}$ is a homogeneous class of minimal degree, and choose $b \in \mathcal{J}$ such that $\phi(a-b)=0$. Then $a-b=c x$ for some $c \in H_{K \times S^{1}}^{*}\left(T^{*} \mathbb{C}^{2 n}\right)$. 
By Proposition 3.5 $c x \in \operatorname{ker} \kappa_{S^{1}} \Rightarrow c \in \operatorname{ker} \kappa_{S^{1}}$, hence $c \in \operatorname{ker} \kappa_{S^{1}} \backslash \mathcal{J}$ is a class of lower degree than $a$.

Corollary 3.8. Let $E$ be an $S^{1}$-equivariant vector bundle on $X$, with an equivariant section $s$ such that the zero set of $s$ is disjoint from the fixed point set $X^{S^{1}}$. Then $e(E)=0 \in H_{S^{1}}^{*}(X)$.

Proof. Consider the bundle $\mathcal{K}^{\oplus 2(n-3)}$ restricted to $X \backslash X^{S^{1}}$. An equivariant section of this bundle is equivalent to an ordinary section of the induced bundle over the quotient $\left(X \backslash X^{S^{1}}\right) / S^{1}$, and by degree considerations we can find a nonvanishing section. Hence $\mathcal{K}^{\oplus 2(n-3)}$ has an equivariant section $t$ over $X$ with zero set supported on $X^{S^{1}}$. Then $s \oplus t$ is a nonvanishing section of $E \oplus \mathcal{K}^{\oplus 2(n-3)}$, hence

$$
0=e\left(E \oplus \mathcal{K}^{\oplus 2(n-3)}\right)=e(E) \cdot e\left(\mathcal{K}^{\oplus 2(n-3)}\right)=x^{2(n-3)} e(E) .
$$

Then by Proposition 3.5, $e(E)=0$.

Remark 3.9. Corollary 3.8 is a weak form of the statement that the restriction map from $H_{S^{1}}^{*}(X)$ to $H_{S^{1}}^{*}\left(X^{S^{1}}\right)$ is injective, proven for compact $X$ in [Ki].

Proof of 3.2. Corollary 3.6 tells us that the characteristic classes $c_{1}, \ldots, c_{n}, p, x$ in $H_{S^{1}}^{*}(X)$ generate the ring, and Corollary 3.7 tells us that it is enough to prove two statements: the first is that $\mathcal{J} \subseteq \operatorname{ker} \kappa_{S^{1}}$, i.e. that the elements of $\mathcal{J}$ are indeed relations in $H_{S^{1}}^{*}(X)$, and the second is that $\phi(\mathcal{J})=\mathcal{I}$. We begin by proving that $\mathcal{J} \subseteq \operatorname{ker} \kappa_{S^{1}}$, following the approach outlined in Remark 3.4

In the nonequivariant context, Konno shows that $\mathcal{E} \cong \mathcal{L}_{i i} \oplus \mathbb{R}$ as a real vector bundle for all $i[\mathrm{~K} 2$. This implies that

$$
p=p_{1}(\mathcal{E})=-c_{2}(\mathcal{E} \otimes \mathbb{C})=-c_{2}\left(\mathcal{L}_{i i} \oplus \mathcal{L}_{i i}^{*}\right)=c_{1}\left(\mathcal{L}_{i i}\right)^{2}=c_{i}^{2} \in H^{*}(X),
$$

and therefore $p-c_{i}^{2} \in$ ker $\kappa$. This argument is adaptable to the equivariant context without any modifications, hence we will not include it here.

Consider the function

$$
\tilde{s}_{i j}: \mu_{\mathbb{R}}^{-1}(\alpha) \cap \mu_{\mathbb{C}}^{-1}(0) \rightarrow \mathbb{C}
$$

given by $\tilde{s}_{i j}(p, q)=\operatorname{det}\left(q_{i} q_{j}\right)$, where $\left(q_{i} q_{j}\right)$ is considered to be a $2 \times 2$ matrix [K2]. This function is $S^{1}$-invariant, and $K$-equivariant with respect to $\rho_{i j}$, and therefore defines an $S^{1}$-equivariant section $s_{i j}$ of $\mathcal{L}_{i j}^{*}$ (the dualization is a consequence of the fact that the action of $K$ on $\mu_{\mathbb{R}}^{-1}(\alpha) \cap \mu_{\mathbb{C}}^{-1}(0)$ is a right action). The vanishing set of $s_{i j}$ is the divisor

$$
Z_{i j}=\left\{[p, q] \in X \mid q_{i} \text { is proportional to } q_{j}\right\},
$$

hence $c_{1}\left(\mathcal{L}_{i j}^{*}\right)=-\frac{1}{2}\left(c_{i}+c_{j}\right)$ is represented in equivariant Borel-Moore homology by the divisor $Z_{i j}$. Now consider the function

$$
\tilde{t}_{i}: \mu_{\mathbb{R}}^{-1}(\alpha) \cap \mu_{\mathbb{C}}^{-1}(0) \rightarrow \mathbb{C}
$$

given by

where

$$
\tilde{t}_{i}(p, q)= \begin{cases}p_{i}^{(2)} / q_{i}^{(1)} & \text { if } q_{i}^{(1)} \neq 0, \\ -p_{i}^{(1)} / q_{i}^{(2)} & \text { if } q_{i}^{(2)} \neq 0,\end{cases}
$$

$$
q_{i}=\left(\begin{array}{c}
q_{i}^{(1)} \\
q_{i}^{(2)}
\end{array}\right) \text { and } p_{i}=\left(p_{i}^{(1)} p_{i}^{(2)}\right)
$$


This function is well defined by the fact that $\mu_{\mathbb{C}}(p, q)=0$. It is $S^{1}$-equivariant with respect to the weight 1 action of $S^{1}$ on $\mathbb{C}$, and it is $K$-equivariant with respect to $\bar{\rho}_{i i}$. Thus it descends to an equivariant section $t_{i}$ of $\mathcal{L}_{i i} \otimes \mathcal{K}$, vanishing on the divisor

$$
W_{i}=\left\{[p, q] \in X \mid p_{i}=0\right\},
$$

so $c_{i}+x=c_{1}\left(\mathcal{L}_{i i} \otimes \mathcal{K}\right)$ is represented by the divisor $W_{i}$.

Consider a nonempty short subset $S \in \mathcal{S}$, and define the vector bundle

$$
E_{S}=\bigoplus_{j \in \overline{S^{c}}} \mathcal{L}_{j n_{S}}^{*} \oplus \bigoplus_{i \in \bar{S}} \mathcal{L}_{i i} \otimes \mathcal{K}
$$

with equivariant Euler class

$$
(-1 / 2)^{\left|\overline{S^{c}}\right|} \prod_{j \in \overline{S^{c}}}\left(c_{j}+c_{n_{S}}\right) \times \prod_{i \in \bar{S}}\left(c_{i}+x\right) .
$$

Then $\left(\bigoplus_{i \in \bar{S}} t_{i}\right) \bigoplus_{j \in \overline{S^{c}}} s_{j n_{S}}$ is a section of $E_{S}$ vanishing only on the cycle

$$
Z_{S}=\bigcap_{i \in \bar{S}} W_{i} \cap \bigcap_{j \in \overline{S^{c}}} Z_{j n_{S}}
$$

consisting of points $[p, q]$ such that $q_{j}$ is proportional to $q_{n_{S}}$ for all $j \in S^{c}$, and $p_{i}=0$ for all $i \in \bar{S}$. We would like to show that $e\left(E_{S}\right)=0 \in H_{S^{1}}^{*}(X)$, and by Corollary 3.8, it will suffice to show that $Z_{S}$ is disjoint from

$$
X^{S^{1}}=M \cup \bigcup_{T \in \mathcal{S}} X_{T} .
$$

Since $S^{c}$ is a long subset that is straight in $Z_{S}$, we have $Z_{S} \cap M=\emptyset$ by Theorem 1.1. We must now show that $Z_{S}$ also does not intersect any $X_{T} \subset X^{S^{1}}$. We begin with the observation that for each $X_{T}, T \in \mathcal{S}^{\prime}$,

$$
[p, q] \in X_{T} \Rightarrow \text { at least two of the vectors in }\left\{p_{i} \mid i \in T\right\} \text { are nonzero. }
$$

This follows from the description of $X_{T}$ given in Theorem 2.1 and the complex moment map conditions. Now let $T \in \mathcal{S}^{\prime}$ be a short subset. If $T \nsubseteq S$, then by the descriptions of $X_{T}$ and $Z_{S}$, we may conclude that any point $[p, q] \in Z_{S} \cap X_{T}$ must have the long subset $T \cup S^{c}$ straight, and $p_{j}=0$ for all $j \in T^{c}$. This means that $(p, q)$ is unstable by Theorem 1.1. so $Z_{S} \cap X_{T}=\emptyset$. On the other hand, if $T \subseteq S$, then we may similarly conclude that for any point $[p, q] \in Z_{S} \cap X_{T}$, the long subset $T^{c}$ is straight, and at most one element in $\left\{p_{i} \mid i \in T\right\}$ is nonzero. This contradicts the observation (2) above, so $Z_{S} \cap X_{T}=\emptyset$.

We have now proven that $\mathcal{J} \subseteq$ ker $\kappa_{S^{1}}$, and it remains only to show that the image $\phi(\mathcal{J})$ is equal to $\mathcal{I}$. In other words (by Theorem 3.1), we must show that the set

$$
\left\{\prod_{j \in \overline{S^{c}}}\left(c_{j}+c_{n_{S}}\right) \times \prod_{i \in \bar{S}} c_{i} \mid \emptyset \neq S \in \mathcal{S}\right\}
$$

spans all monomials of degree $2(n-2)$ in the ring

$$
\mathbb{Q}\left[c_{1}, \ldots, c_{n}\right] /\left\langle c_{i}^{2}-c_{1}^{2} \mid i \in\{2, \ldots, n\}\right\rangle .
$$


Let $b_{k}=\frac{1}{2}\left(c_{1}+c_{k}\right)$ for all $k$, so that $c_{k}=2 b_{k}-b_{1}$. The relations $c_{k}^{2}=c_{1}^{2}$ translate to $b_{k}^{2}=b_{1} b_{k}$ for all $k$. Let

$$
v_{S}=\frac{(-1)^{n}}{2^{\left|\overline{S^{c}}\right|}} \prod_{j \in \overline{S^{c}}}\left(c_{j}+c_{n_{S}}\right) \times \prod_{i \in \bar{S}} c_{i}=(-1)^{n} \prod_{j \in \overline{S^{c}}}\left(b_{j}+b_{n_{S}}-b_{1}\right) \times \prod_{i \in \bar{S}}\left(2 b_{i}-b_{1}\right),
$$

and let

$$
b_{A}=(-1)^{|A|} b_{1}^{n-2-|A|} \prod_{k \in A} b_{k}
$$

for all $A \subsetneq\{2, \ldots, n\}$. Then $\left\{b_{A}\right\}$ is a basis for the $(n-2)^{\text {nd }}$ graded piece of the ring

$$
\mathbb{Q}\left[b_{1}, \ldots, b_{n}\right] /\left\langle b_{k}^{2}-b_{1} b_{k} \mid k \in\{2, \ldots, n\}\right\rangle,
$$

hence we need to show that each element $b_{A}$ can be expressed as a linear combination of the elements $\left\{v_{S} \mid S \in \mathcal{S}\right\}$.

Claim 3.10. We have the following expression for $v_{S}$ in terms of the basis $\left\{b_{A}\right\}$ :

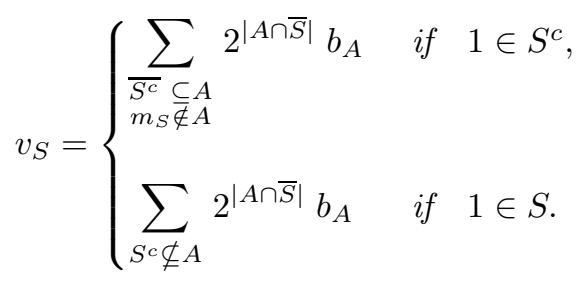

Proof. Any degree $n-2$ monomial in $b_{1}, \ldots, b_{n}$ is equal to $(-1)^{|A|} b_{A}$, where $A$ is the set of $k>1$ such that $b_{k}$ appears in the monomial. Expanding $v_{S}$, we need to count (with sign) the occurrence of $b_{A}$ for each $A$. In most cases we find that there is no cancellation, and the claim is straightforward. The most difficult case occurs when $1 \in S$ (therefore $n_{S}=1$ ) and $n_{S} \in A$; in this case the number of times (with multiplicity) that $b_{A}$ occurs in $v_{S}$ is

$$
\begin{aligned}
& (-1)^{n}(-1)^{|A|}(-1)^{\left|A^{c} \cap \bar{S}\right|} 2^{|A \cap \bar{S}|} \sum_{E \subsetneq A^{c} \cap \overline{S^{c}}}(-1)^{|E|} \\
= & (-1)^{n}(-1)^{|A|}(-1)^{\left|A^{c} \cap \bar{S}\right|} 2^{|A \cap \bar{S}|}\left((1-1)^{\left|A^{c} \cap \overline{S^{c}}\right|}-(-1)^{\left|A^{c} \cap \overline{S^{c}}\right|}\right) \\
= & (-1)^{n+|A|+\left|A^{c} \cap \bar{S}\right|+\left|A^{c} \cap \overline{S^{c}}\right|+1} 2^{|A \cap \bar{S}|} \\
= & (-1)^{2 n} 2^{|A \cap \bar{S}|} \\
= & 2^{|A \cap \bar{S}|}
\end{aligned}
$$

(When we write $A^{c}$, we mean the complement of $A$ inside of $\{2, \ldots, n\}$.) We leave the remaining cases to be checked by the reader.

Claim 3.11. Suppose that $1 \in S$. Let $S_{0}=S$, and for $1 \leq k \leq|S|$, define the set $S_{k}:=S_{k-1} \backslash\left\{m_{S_{k-1}}\right\}$ (i.e. $S_{k}$ consists of the $|S|-k$ largest elements of $S$ ). Then $v_{S}+\sum_{k=1}^{|S|-1} 2^{k-1} v_{S_{k}}=\sum_{A} 2^{|A \cap \bar{S}|} b_{A}$.

Proof. We proceed by induction to show that

$$
v_{S}+\sum_{k=1}^{l} 2^{k-1} v_{S_{k}}=\sum_{A} 2^{|A \cap \bar{S}|} b_{A}-2^{l} \sum_{S_{l+1}^{c} \subseteq A} 2^{\left|A \cap \bar{S}_{l}\right|} b_{A} ;
$$


the case $l=|S|-1$ is the statement of the claim. The base case $l=0$ follows from Claim 3.10, together with the observation that $\overline{S_{1}^{c}}=S^{c}$. More generally, for all $l \geq 1$, we have $\overline{S_{l+1}^{c}}=S^{c} \cup\left\{m_{S_{1}}, \ldots, m_{S_{l}}\right\}$. Then

$$
\begin{aligned}
v_{S}+\sum_{k=1}^{l+1} 2^{k-1} v_{S_{k}} & =v_{S}+\sum_{k=1}^{l} 2^{k-1} v_{S_{k}}+2^{l} v_{S_{l+1}} \\
& =\sum_{A} 2^{|A \cap \bar{S}|} b_{A}-2^{l} \sum_{\frac{\sum_{S_{l+1}^{c}} \subseteq A}{} 2^{\left|A \cap \bar{S}_{l}\right|} b_{A}+2^{l} \sum_{\substack{S_{l+1}^{c} \subseteq A \\
m_{S_{l+1}} \notin A}} 2^{\left|A \cap \bar{S}_{l+1}\right|} b_{A}}
\end{aligned}
$$

by the inductive hypothesis and Claim 3.10. Using the fact that $A \cap \bar{S}_{l+1}=A \cap \bar{S}_{l}$ when $m_{S_{l+1}} \notin A$, this is equal to

$$
\sum_{A} 2^{|A \cap \bar{S}|} b_{A}-2^{l} \sum_{\overline{S_{l+1}^{c}} \cup\left\{m_{S_{l+1}}\right\} \subseteq A} 2^{\left|A \cap \bar{S}_{l}\right|} .
$$

Finally, since $\left|A \cap \bar{S}_{l+1}\right|=\left|A \cap \bar{S}_{l}\right|-1$ when $m_{S_{l+1}} \in A$, this reduces to

$$
\sum_{A} 2^{|A \cap \bar{S}|} b_{A}-2^{l+1} \sum_{\overline{S_{l+2}^{c}} \subseteq A} 2^{\left|A \cap \bar{S}_{l+1}\right|} b_{A},
$$

thus proving our claim.

For all short subsets $T$ containing 1 , let $w_{T}=\sum_{A} 2^{|A \cap \bar{T}|} b_{A}$, which by Claim 3.11 is expressible as a linear combination of elements of the set $\left\{v_{S} \mid \emptyset \neq S \in \mathcal{S}\right\}$. Let

$$
x_{S}= \begin{cases}\sum_{1 \in T \subseteq S}(-1)^{|S|+|T|} w_{T} & \text { if } 1 \in S, \\ v_{S} & \text { if } 1 \in S^{c} .\end{cases}
$$

Our last task will be to prove that the transition matrix $Q$ taking the basis $\left\{b_{A}\right\}$ to the set $\left\{x_{S}\right\}$ is upper triangular with ones on the diagonal, and therefore invertible. In order to make sense of "the diagonal," we must first give an explicit bijection between the set of proper subsets of $\{2, \ldots, n\}$ and the set of nonempty short subsets of $\{1, \ldots, n\}$. We do this as follows: given $A \subsetneq\{2, \ldots, n\}$, let

$$
S(A)= \begin{cases}A^{c} & \text { if } A^{c} \text { is short, } \\ \{1, \ldots, n\} \backslash A^{c}=A \cup\{1\} & \text { if } A^{c} \text { is long. }\end{cases}
$$

The rows of $Q$ will be indexed by $A$, and the sets will appear in lexicographic order within cardinality class. For example, when $n=4$, the order of the rows will be $\emptyset$, $\{2\},\{3\},\{4\},\{2,3\},\{2,4\},\{3,4\}$. The columns will be indexed by $S$ according to the bijection described above.

Claim 3.12. The matrix $Q$ is lower triangular with ones on the diagonal.

Proof. First consider a column corresponding to a short subset $S$ that does not contain 1. The entries in this column correspond to the coefficient of $b_{A}$ in $x_{S}=v_{S}$. From Claim 3.10, we see that $b_{A}$ appears in $v_{S}$ only if $\overline{S^{c}} \subseteq A \subseteq \overline{S^{c}} \cup \bar{S}$, and if so it appears with a coefficient of $2^{|A \cap \bar{S}|}$. Since $1 \notin S$, we have

$$
\overline{S^{c}}=S^{c} \backslash\{1\}=\{2, \ldots, n\} \backslash S .
$$


The diagonal entry corresponds to the set $A=\{2, \ldots, n\} \backslash S=\overline{S^{c}}$, therefore in this row we get the number $2^{|A \cap \bar{S}|}=2^{\left|\overline{S^{c}} \cap \bar{S}\right|}=1$. Since the set $A$ corresponding to a given row can never contain the set $B$ corresponding to a lower row, the rows above the diagonal fail to satisfy the condition $\overline{S^{c}} \subseteq A$, and we get all zeros.

Now consider a column corresponding to a short subset $S$ that does contain 1. In this case, the coefficient of $b_{A}$ in $x_{S}$ is

$$
(-1)^{|S|} \sum_{1 \in T \subseteq S}(-1)^{|T|} 2^{|A \cap \bar{T}|} .
$$

The diagonal entry corresponds to the set $A=\bar{S}$, and we get

$$
\begin{aligned}
(-1)^{|S|} \sum_{1 \in T \subseteq S}(-1)^{|T|} 2^{|\bar{T}|} & =(-1)^{|\bar{S}|} \sum_{1 \in T \subseteq S}(-2)^{|\bar{T}|} \\
& =(-1)^{|S|}(1-2)^{|\bar{S}|}=1 .
\end{aligned}
$$

Any row above the diagonal corresponds to a set $A$ which does not contain $\bar{S}$. Choose an element $l \in \bar{S} \backslash A$. Then

$$
\begin{aligned}
(-1)^{|S|} \sum_{1 \in T \subseteq S}(-1)^{|T|} 2^{|A \cap \bar{T}|} & =(-1)^{|S|} \sum_{l \in T}(-1)^{|T|} 2^{|A \cap \bar{T}|}+(-1)^{|S|} \sum_{l \notin T}(-1)^{|T|} 2^{|A \cap \bar{T}|} \\
& =(-1)^{|S|} \sum_{l \notin T}\left[(-1)^{|T|} 2^{|A \cap \bar{T}|}+(-1)^{|T \cup\{l\}|} 2^{|A \cap \bar{T}|}\right] \\
& =0 .
\end{aligned}
$$

Claim 3.12 tells us that each $b_{A}$ can be expressed as a linear combination of elements of the form $x_{S}$, and therefore of elements of the form $v_{S}$. This lets us conclude that $\phi(\mathcal{J})=\mathcal{I}$, and thereby completes the proof of Theorem 3.2

\section{The COHOMOLOGY RING OF A CORE COMPONENT}

In this section we compute the $S^{1}$-equivariant and ordinary cohomology rings of the core component $U_{S}$ corresponding to a short subset $S \subseteq\{1, \ldots, n\}$. Since $U_{S}$ is the closure of a cell in an even-dimensional equivariant cellular decomposition of $X$, the restriction map $H_{S^{1}}^{*}(X) \rightarrow H_{S^{1}}^{*}\left(U_{S}\right)$ is surjective. In particular, $H_{S^{1}}^{*}\left(U_{S}\right)$ is generated by restrictions of the Kirwan classes $c_{1}, \ldots, c_{n}, x$. For our presentation, it will be convenient to assume that $1 \in S$, and to work with the classes $b_{k}=\frac{1}{2}\left(c_{1}+c_{k}\right)$ introduced in Section 3 . With respect to these generators, we obtain the following result.

Theorem 4.1. The equivariant cohomology ring $H_{S^{1}}^{*}\left(U_{S}\right)$ is isomorphic to $\mathbb{Q}\left[b_{1}, \ldots, b_{n}, x\right] / \mathcal{J}_{S}$, where $\mathcal{J}_{S}$ is generated by the following four families:

1) $b_{1}-b_{i}$ for all $i \in S$,

2) $b_{j}\left(b_{1}-b_{j}\right)$ for all $j \in S^{c}$,

$3 \prod_{j \in R} b_{j}$ for all $R \subseteq S^{c}$ such that $R \cup S$ is long,

4) $h s\left(b_{1}+x\right)^{|S|-1} \cdot \frac{1}{b_{1}}\left(\prod_{j \in L}\left(b_{j}-b_{1}\right)-\prod_{j \in L} b_{j}\right)$ for all long subsets $L \subseteq S^{c}$.

Corollary 4.2. The ordinary cohomology ring $H^{*}\left(U_{S}\right)$ is isomorphic to $\mathbb{Q}\left[b_{1}, \ldots, b_{n}\right] / \mathcal{I}_{S}$, where $\mathcal{I}_{S}$ is generated by the following four families:

1) $b_{1}-b_{i}$ for all $i \in S$, 
2) $b_{j}\left(b_{1}-b_{j}\right)$ for all $j \in S^{c}$,

3) $\prod_{j \in R} b_{j}$ for all $R \subseteq S^{c}$ such that $R \cup S$ is long,

4) $b_{1}^{|S|-2} \prod_{j \in L}\left(b_{j}-b_{1}\right) \quad$ for all long subsets $L \subseteq S^{c}$.

Remark 4.3. Each of these relations has a geometric interpretation. For $i \in\{1, \ldots, n\}$, it is possible to construct a line bundle on $X$ with equivariant Euler class $b_{i}-b_{1}$ which has a section supported on the locus, where $q_{1} q_{1}^{*}$ and $q_{i} q_{i}^{*} \in \mathbb{R}^{3}$ point in opposite directions. Since this locus is disjoint from $U_{S}$ when $i \in S$, we have

$$
\text { 1) } b_{i}=b_{1} \in H_{S^{1}}^{*}\left(U_{S}\right) \text { for all } i \in S .
$$

Similarly, we showed in the proof of Theorem 3.2 that $-b_{j}=-\frac{1}{2}\left(c_{1}+c_{j}\right)$ is represented by the divisor $Z_{1 j}$ on which $q_{1} q_{1}^{*}$ and $q_{i} q_{i}^{*} \in \mathbb{R}^{3}$ point in the same direction. Then by the previous reasoning, we obtain

$$
\text { 2) } b_{j}\left(b_{1}-b_{j}\right)=0 \in H_{S^{1}}^{*}\left(U_{S}\right) \text { for all } j \in S^{c} .
$$

Recall from Section 3 that for any $R \subseteq S^{c}$, the cohomology class $(-1)^{|R|} \prod_{j \in R} b_{j}$ is represented by the subvariety $Z_{R} \subseteq X$ of points with $q_{j}$ proportional to $q_{1}$ for all $j \in R$. When restricted to $U_{S}$, this becomes $U_{S} \cap U_{R \cup S}$, the unstable manifold for the critical locus $X_{R \cup S} \cap U_{S}$ of the Morse-Bott function $\left.\Phi\right|_{U_{S}}$. In particular, we have

$$
\text { 3) } \prod_{j \in R} b_{j}=0 \in H_{S^{1}}^{*}\left(U_{S}\right) \text { if } R \cup S \text { is long. }
$$

To understand the fourth family of relations, recall from Section 3 that

$$
b_{1}+x=2 b_{i}-b_{1}+x=c_{i}+x \in H_{S^{1}}^{*}\left(U_{S}\right)
$$

is represented by the divisor $W_{i}$ of points with $p_{i}=0$ for any $i \in S$. In particular, the class $\left(b_{1}+x\right)^{|S|-1}$ is represented by the subvariety of points in $U_{S}$ on which $p_{i}=0$ for all $i \in \bar{S}$, which is equal to $M_{S}$ by the complex moment map condition. Hence the fourth family of generators of $\mathcal{J}_{S}$ (or of $\mathcal{I}_{S}$ ) can be interpreted geometrically as $\left(b_{1}+x\right)^{|S|-1}$ (respectively $b_{1}^{|S|-1}$ in the nonequivariant case) times classes that vanish in $H_{S^{1}}^{*}\left(M_{S}\right)$ (see Lemma 4.5).

Proof of $\left[4.1\right.$. Let $\phi: \mathbb{Q}\left[b_{1}, \ldots, b_{n}, x\right] \rightarrow H_{S^{1}}^{*}\left(U_{S}\right)$ denote the composition of the Kirwan map with restriction to $U_{S}$. Our claim is that $\operatorname{ker} \phi=\mathcal{J}_{S}$. For every short subset $T$ containing $S$, let

$$
\phi_{T}: \mathbb{Q}\left[b_{1}, \ldots, b_{n}, x\right] \rightarrow H_{S^{1}}^{*}\left(X_{T} \cap U_{S}\right)
$$

denote the composition of the Kirwan map with restriction to $X_{T} \cap U_{S}$, and let

$$
J_{T}=\operatorname{ker} \phi_{T} .
$$

Similarly, let

$$
\phi_{\emptyset}: \mathbb{Q}\left[b_{1}, \ldots, b_{n}, x\right] \rightarrow H_{S^{1}}^{*}\left(M_{S}\right)
$$

be the natural map, and let

$$
J_{\emptyset}=\operatorname{ker} \phi_{\emptyset} .
$$

The kernel of the restriction map $H_{S^{1}}^{*}\left(U_{S}\right) \rightarrow H_{S^{1}}^{*}\left(U_{S}^{S^{1}}\right)$ to the fixed point set of $U_{S}$ is a torsion module over $H_{S^{1}}^{*}(p t)$ [AB 3.5], and Proposition 3.5 tells us that $H_{S^{1}}^{*}\left(U_{S}\right)$ is a free $H_{S^{1}}^{*}(p t)$-module. Hence the restriction map is injective, and we have

$$
\operatorname{ker} \phi=\operatorname{ker} \phi_{\emptyset} \cap \bigcap_{T \supseteq S} \operatorname{ker} \phi_{T} .
$$


We know that $X_{T} \cap U_{S} \cong \mathbb{C} P^{|S|-2}$ for all short $T \supseteq S$; therefore

$$
H_{S^{1}}^{*}\left(X_{T} \cap U_{S}\right) \cong \mathbb{Q}[h, x] / h^{|S|-1} .
$$

Furthermore, we know that for all $i \in T$, the restriction of $b_{i}+x$ to $H_{S^{1}}^{*}\left(U_{T}\right)$ is represented by the divisor $W_{i} \cap U_{T}$ (see Remark 4.3), and therefore restricts to the class of a hyperplane on $X_{T} \cap U_{S}$. Hence $\phi_{T}\left(b_{i}+x\right)=h$ for all $i \in T$. On the other hand, for $j \in T^{c}$, the class $b_{j}$ is represented by the divisor $Z_{1 j}$ on $X$, which is disjoint from $X_{T} \cap U_{S}$, hence $\phi\left(b_{j}\right)=0$ for all $j \in T^{c}$. Thus we conclude that

$$
\operatorname{ker} \phi_{T}=\left\langle b_{1}-b_{i}, b_{j},\left(b_{1}+x\right)^{|S|-1} \mid i \in T, j \in T^{c}\right\rangle .
$$

Lemma 4.4. The intersection $\bigcap_{T \supseteq S} \operatorname{ker} \phi_{T}$ is equal to

$$
\left\langle\begin{array}{l|l}
b_{1}-b_{i}, b_{j}\left(b_{1}-b_{j}\right), \prod_{j \in R} b_{j},\left(b_{1}+x\right)^{|S|-1} & \begin{array}{l}
i \in S, j \in S^{c} \\
R \cup S \text { long }
\end{array}
\end{array}\right\rangle .
$$

Proof. First, since the variable $x$ appears only in the generator $\left(b_{1}+x\right)^{|S|-1}$, which is contained in every ideal on both sides of the equation, we may reduce the problem to showing that

$$
\begin{aligned}
& \bigcap_{T \supseteq S}\left\langle b_{1}-b_{i}, b_{j} \mid i \in T, j \in T^{c}\right\rangle \\
& =\left\langle\begin{array}{l|l}
b_{1}-b_{i}, b_{j}\left(b_{1}-b_{j}\right), \prod_{j \in R} b_{j} & \begin{array}{l}
i \in S, j \in S^{c} \\
R \cup S \text { long }
\end{array}
\end{array}\right\rangle
\end{aligned}
$$

in the ring $\mathbb{Q}\left[b_{1}, \ldots, b_{n}\right]$. Both ideals cut out the (reducible) variety

$$
\bigcup_{T \supseteq S} Y_{T} \subseteq \operatorname{Spec} \mathbb{Q}\left[b_{1}, \ldots, b_{n}\right],
$$

where

$$
Y_{T}=\left\{\left(z_{1}, \ldots, z_{n} \mid z_{i}=z_{1} \forall i \in S, z_{j}=0 \forall j \in S^{c}\right\} .\right.
$$

The left-hand side of (3) is an intersection of prime ideals, and is therefore radical. Thus by Hilbert's Nullstellensatz, it is sufficient to prove that the right-hand side of (3) is radical. This involves showing that the ideal is saturated, with Hilbert polynomial equal to the constant \#\{short $T \supseteq S\}$.

The degree $k$ piece of the quotient

$$
\mathbb{Q}\left[b_{1}, \ldots, b_{n}\right] /\left\langle b_{1}-b_{i}, b_{j}\left(b_{1}-b_{j}\right) \mid i \in S, j \in S^{c}\right\rangle
$$

has a basis of elements of the form

$$
b_{1}^{e_{1}} \prod_{j \in S^{c}} b_{j}^{e_{j}},
$$

where $e_{j} \in\{0,1\}$ for all $j>0$, and $e_{1}+\sum_{j \in S^{c}} e_{j}=k$. The subset of these elements with the property that $S \cup\left\{j \mid e_{j}=1\right\}$ is short descends to a basis for the degree $k$ part of the ring

$$
\left.\mathbb{Q}\left[b_{1}, \ldots, b_{n}\right] /\left\langle b_{1}-b_{i}, b_{j}\left(b_{1}-b_{j}\right), \prod_{j \in R} b_{j}\right| \begin{array}{c}
i \in S, j \in S^{c} \\
R \cup S \text { long }
\end{array}\right),
$$

hence our ideal has the desired Hilbert polynomial. It is also clear from this description that if an element $a$ of the quotient ring is nonzero, so is $b_{1}^{d} \cdot a$ for any $d \geq 0$, hence our ideal is saturated. 
It now remains to show that

$$
\mathcal{J}_{S}=\left\langle b_{1}-b_{i}, b_{j}\left(b_{1}-b_{j}\right), \prod_{j \in R} b_{j},\left(b_{1}+x\right)^{|S|-1} \mid \begin{array}{l}
i \in S, j \in S^{c} \\
R \cup S \text { long }
\end{array}\right\rangle \cap \operatorname{ker} \phi_{\emptyset} .
$$

The fact that $\mathcal{J}_{S}$ is contained in the intersection is clear. To show the opposite containment, consider an element

$a+\eta \cdot\left(b_{1}+x\right)^{|S|-1} \in\left\langle\begin{array}{l|l}b_{1}-b_{i}, b_{j}\left(b_{1}-b_{j}\right), \prod_{j \in R} b_{j},\left(b_{1}+x\right)^{|S|-1} & \begin{array}{c}i \in S, j \in S^{c} \\ R \cup S \text { long }\end{array}\end{array},\right.$,

with

$$
a \in\left\langle\begin{array}{l|l}
b_{1}-b_{i}, b_{j}\left(b_{1}-b_{j}\right), \prod_{j \in R} b_{j} & \begin{array}{c}
i \in S, j \in S^{c} \\
R \cup S \text { long }
\end{array}
\end{array}\right\rangle,
$$

and suppose that we also have

$$
a+\eta \cdot\left(b_{1}+x\right)^{|S|-1} \in \operatorname{ker} \phi_{\emptyset} .
$$

Lemma 4.5 ([HK2]). The kernel of $\phi_{\emptyset}$ is equal to

$$
\left\langle b_{1}-b_{i}, b_{j}\left(b_{1}-b_{j}\right), \prod_{j \in R} b_{j},\left(b_{1}+x\right)^{|S|-1} b_{1}^{-1}\left(\prod_{j \in L}\left(b_{j}-b_{1}\right)-\prod_{j \in L} b_{j}\right)\right\rangle,
$$

where $i \in S, j \in S^{c}$, and $R, L \subseteq S^{c}$, with $R \cup S$ and $L$ both long.

Lemma 4.5 tells us that $a \in \operatorname{ker} \phi_{\emptyset}$; therefore

$$
\eta \cdot\left(b_{1}+x\right)^{|S|-1} \in \operatorname{ker} \phi \emptyset \cdot
$$

However, $\left(b_{1}+x\right)^{|S|-1}$ is represented in $H_{S^{1}}^{*}\left(U_{S}\right)$ by the subvariety $M_{S}$ (see Remark 4.3), hence

$$
0=\phi_{\emptyset}\left(\eta \cdot\left(b_{1}+x\right)^{|S|-1}\right)=\phi_{\emptyset}(\eta) \cdot e\left(M_{S}\right),
$$

where $e\left(M_{S}\right)$ is the equivariant Euler class of the normal bundle to $M_{S}$ inside of $U_{S}$. Since the equivariant Euler class of the normal bundle to a component of the fixed point set is never a zero-divisor, we have $\eta \in \operatorname{ker} \phi_{\emptyset}$. Then by Lemma 4.5,

$$
a+\eta \cdot\left(b_{1}+x\right)^{|S|-1} \in \mathcal{J}_{S} .
$$

This completes the proof of Theorem 4.1

Example 4.6. For arbitrary $n$ and $\alpha$, suppose that $S$ is a maximal short subset. Then Corollary 4.2 tells us that $H^{*}\left(U_{S}\right) \cong \mathbb{Q}\left[b_{1}\right] /\left\langle b_{1}^{n-2}\right\rangle$. We conjecture that in this case we in fact have $U_{S} \cong \mathbb{C} P^{n-3}$.

Example 4.7. Consider the core component pictured in Example 2.3. By Theorem 4.2 and Remark 4.3,

$$
\begin{aligned}
& H^{*}\left(U_{S}\right) \\
\cong & \mathbb{Q}\left[b_{1}, b_{3}, b_{4}, b_{5}\right] /\left\langle\begin{array}{c}
b_{3}\left(b_{1}-b_{3}\right), b_{4}\left(b_{1}-b_{4}\right), b_{5}\left(b_{1}-b_{5}\right), b_{3} b_{4}, b_{3} b_{5}, b_{4} b_{5}, \\
b_{1}\left(b_{1}-b_{3}-b_{4}\right), b_{1}\left(b_{1}-b_{3}-b_{5}\right), b_{1}\left(b_{1}-b_{4}-b_{5}\right)
\end{array}\right\rangle,
\end{aligned}
$$

where $b_{1}$ is the fundamental class of $M_{S}$, and $b_{3}, b_{4}$, and $b_{5}$ are the negatives of the fundamental classes of the curves labeled 123, 124, and 125, respectively. 
Because the transverse intersection of two complex varieties is positive, we know that $-b_{1} b_{3}\left[U_{S}\right]=1$. With respect to the basis

$$
\left\{b_{1}-b_{3}-b_{4}-b_{5}, b_{3}, b_{4}, b_{5}\right\},
$$

the intersection form on $H^{2}\left(U_{S}\right)$ is represented by the matrix

$$
\left(\begin{array}{llll}
1 & & & \\
& -1 & & \\
& & -1 & \\
& & & -1
\end{array}\right) \text {. }
$$

Hence $U_{S}$ is homeomorphic to the blow-up of $\mathbb{C} P^{2}$ at three points.

Example 4.8. Using the same $\alpha=0 \oplus(1,1,3,3,3)$, consider the short subset $S=\{1,3\}$. In this case, Theorem 4.2 tells us that

$$
H^{*}\left(U_{S}\right) \cong \mathbb{Q}\left[b_{1}, b_{2}\right] /\left\langle b_{1}^{2}, b_{2}\left(b_{1}-b_{2}\right)\right\rangle .
$$

With respect to the basis $\left\{b_{1}-b_{2}, b_{2}\right\}$, the intersection form on $H^{2}\left(U_{S}\right)$ is repre-

sented by the matrix $\left(\begin{array}{cc}-1 & 0 \\ 0 & 1\end{array}\right)$, hence $U_{S}$ is homeomorphic to the blow-up of $\mathbb{C} P^{2}$ at a single point.

\section{ACKNOWLEDGMENTS}

We are grateful to Allen Knutson for guiding us through the polygonal world, to Tamás Hausel for introducing us to the work of Konno, and also to Michael Thaddeus for useful discussions. The second author thanks the Swiss National Funds for Scientific Research for its support.

\section{REFERENCES}

[AB] M. Atiyah and R. Bott. The moment map and equivariant cohomology. Topology 23 (1984) no. 1, 1-28. MR 85e:58041

[BD] R. Bielawski and A. Dancer. The geometry and topology of toric hyperkähler manifolds. Comm. Anal. Geom. 8 (2000), 727-760. MR 2002c:53078]

[HP] M. Harada and N. Proudfoot. Properties of the residual circle action on a hypertoric variety. To appear in Pacific Journal of Mathematics.

[HS] T. Hausel and B. Sturmfels. Toric hyperkähler varieties. math.AG/0203096.

[HK1] J-C. Hausmann, A. Knutson. Polygon spaces and Grassmannians. L'Enseignement Mathématique. 43 (1997), 173-198. MR 98e:58035

[HK2] J-C. Hausmann, A. Knutson. The cohomology ring of polygon spaces. Ann. Inst. Fourier, Grenoble 48 (1998), no. 1, 281-321. MR 99a:58027

[Ki] F. Kirwan. Cohomology of quotients in symplectic and algebraic geometry. Mathematical Notes 31, Princeton University Press, 1984. MR 86i:58050

[Kl] A. Klyachko. Spatial polygons and stable configurations of points in the projective line. Algebraic geometry and its applications (Yaroslavl, 1992), Aspects Math., Vieweg, Braunschweig (1994), 67-84. MR 95k:14015

[K1] H. Konno. Cohomology rings of toric hyperkähler manifolds. Internat. J. Math. 11 (2000), no. 8, 1001-1026. MR 2001k:53089

[K2] H. Konno. On the cohomology ring of the HyperKähler analogue of the Polygon Spaces. Integrable systems, topology, and physics (Tokyo, 2000), 129-149, Contemp. Math., 309, Amer. Math. Soc., Providence, RI, 2002. MR 2003k:53111

[N1] H. Nakajima. Instantons on ALE spaces, quiver varieties, and Kac-Moody algebras. Duke Mathematical Journal 72 (1994) no. 2, 365-416. MR 95i:53051.

[N2] H. Nakajima. Varieties associated with quivers. Canadian Math. Soc. Conf. Proc. 19 (1996), 139-157. MR 97m:16022 
[N3] H. Nakajima. Quiver varieties and Kac-Moody algebras. Duke Math. J. 91 (1998), no. 3, 515-560. MR 99b:17033

[N4] H. Nakajima. Quiver varieties and finite dimensional representations of quantum affine algebras. J. Amer. Math. Soc. 14 (2001), no. 1, 145-238 (electronic). MR 2002i:17023

Department of Mathematics, University of Toronto, Ontario, Canada M5S 3G3

E-mail address: megumi@math.toronto.edu

Department of Mathematics, University of California, Berkeley, California 94720

E-mail address: proudf@math.berkeley.edu 